\title{
Diagnosing the most common odontogenic cystic and osseous lesions of the jaws for the practicing pathologist
}

\author{
Robert A Robinson \\ Department of Pathology, University of Iowa, Iowa City, IA, USA
}

\begin{abstract}
Odontogenic cysts and osseous lesions are often seen as challenging diagnostic lesions but the majority of them are easily classified. This article outlines the diagnostic features required for separating the most common of odontogenic cysts and select osseous lesions of the jaws. Clinical and radiographic findings of these jaw lesions often lead to a differential diagnosis that only the histologic findings will clarify. Dentigerous cyst, keratocystic odontogenic tumor, and certain ameloblastomas that have cystic change, may have identical radiographic findings, with only separation by their specific histologic features leading to the significantly different treatments required for each. Conversely, some cystic lesions can appear histologically identical and cannot be diagnosed without the radiographic findings. Certain osseous lesions of the jaws are particularly problematic for diagnosis without the appropriate radiographic findings, and the diagnosis should probably not be attempted on the histologic findings alone. This article will integrate the necessary clinical, radiographic, and histologic findings required to address the most common odontogenic lesions.

Modern Pathology (2017) 30, S96-S103; doi:10.1038/modpathol.2016.191
\end{abstract}

Odontogenic cysts and osseous lesions may not be commonly encountered in the daily practice of many pathologists. This makes their diagnosis seem more challenging as there is little practice in understanding the features required for their classification. However, the diagnosis of the vast majority of these lesions is not difficult. A few key histologic observations are all that are required in many cases. But the diagnosis of odontogenic lesions also requires very basic knowledge of the clinical, and especially the radiographic, features with which they are associated. ${ }^{1}$ In this regard, the manner of diagnosis of odontogenic lesions is analogous to that of bone tumors, where understanding the radiographic findings is critical. This article describes some of the most common odontogenic cysts and osseous lesions and outlines the steps to take in establishing the correct diagnosis.

\section{Odontogenic cystic lesions}

Basic Radiographic Considerations the Pathologist Needs to Know About Cystic Odontogenic Lesions

The pathologist should first understand cystic odontogenic lesions from the perspective of the oral

Correspondence: Dr RA Robinson, $\mathrm{MD}, \mathrm{PhD}$, Department of Pathology, 200 Hawkins Drive, University of Iowa, Iowa City, IA 52242, USA.

E-mail: robert-a-robinson@uiowa.edu

Received 24 May 2016; revised 6 September 2016; accepted 14 September 2016 surgeon and dentist. As an example, an impacted third molar is seen on the radiograph in Figure 1. Radiographically this radiolucency could represent dentigerous cyst, keratocystic odontogenic tumor, unicystic ameloblastoma, and ameloblastoma with cystic change. In this situation, the initial steps of treatment would involve removing the tooth and curetting the associated tissues. The associated soft tissue should be sent for histologic evaluation. Depending on the histologic diagnosis, the patient may (1) require no further treatment other than the simple enucleation of the cyst already performed in the case of dentigerous cyst, (2) secondary surgical treatment with mandatory long-term radiographic follow-up in the case of keratocystic odontogenic tumor or unicystic ameloblastoma, or (3) even major jaw excision/resection in the case of ameloblastoma. On the other hand, in some cystic lesions, such as radicular cyst, the final diagnosis cannot be made without a radiographic image or an accurate intraoperative description in association with the histologic appearance. The key radiographic features of the entities discussed in this article will be discussed with each.

\section{Keratocystic Odontogenic Tumor}

Keratocystic odontogenic tumor is not the most common of odontogenic cysts but it is an extremely important one for the pathologist to recognize. It is a 


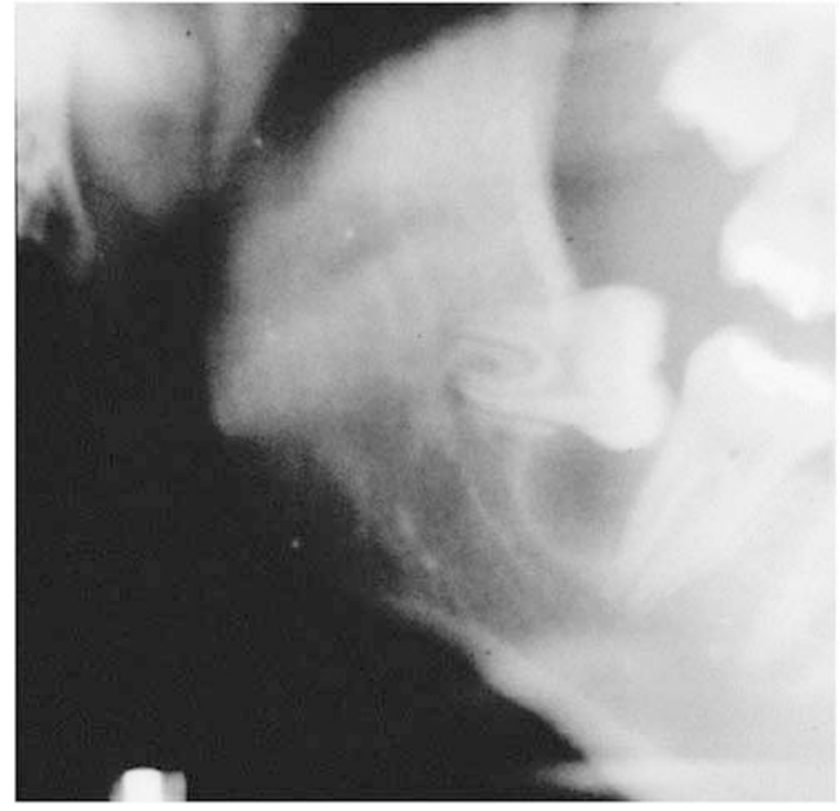

Figure 1 Impacted molar tooth with associated well-corticated and circumscribed radioluceny. The radiographic differential includes, among others, dentigerous cyst, keratocystic odontogenic tumor, unicystic ameloblastoma, and ameloblastoma with cystic change. The histologic findings of the cyst lining are required for diagnosis.

diagnosis always on the mind of dentists and oral surgeons when they encounter a cystic lesion of the jaw. This cystic neoplasm is thought to arise from the residual odontogenic epithelial rests of the dental lamina in the jaw that remain after tooth formation.

For many years keratocystic odontogenic tumor was named parakeratinizing odontogenic keratocyst, with no reference to tumor or neoplasm. However, clinicians and pathologists alike knew that this 'cyst' would recur if it were simply curetted and that this histologically benign process could grow so large as to involve the entire mandible or maxilla. ${ }^{2,3}$ It was demonstrated in 2004 that keratocystic odontogenic tumors exhibited loss of heterozygosity in the epithelial cells of the cyst lining, pointing to these lesions as being neoplastic, rather than being developmental cysts. ${ }^{4,5}$

Further intriguing insights to the origin of keratocystic odontogenic tumor comes from the investigation of patients with nevoid basal cell carcinoma syndrome (Gorlin syndrome), a syndrome in which keratocystic odontogenic tumors are very common. ${ }^{6}$ These studies have shown that the genetic abnormalities seen in these patients were found to be associated with patched 1 (PTCH1) gene mutations (9q22.3). As the syndrome's name implies, basal cell carcinoma, usually multiple and occurring at an early age, is a major diagnostic criterion for the syndrome, along with keratocystic odontogenic tumor. Other major diagnostic criteria are palmar or plantar skin pitting, calcification of the falx cerebri and medulloblastoma. Many other findings, considered as minor criteria, are found in these patients, including bifid ribs, fibromas of the ovary or heart and ocular changes. ${ }^{7,8}$ The majority of patients that most pathologists will encounter that have keratocystic odontogenic tumor will not be in patients with nevoid basal cell carcinoma syndrome, ie, they are sporadic cases. Interestingly perhaps only $50 \%$ of patients with sporadic keratocystic odontogenic tumor have abnormalities of PTCH1 gene. ${ }^{9}$ As patients with basal cell nevus syndrome often have multiple keratocystic odontogenic tumors, it is important for the pathologist to remember that in any patient with more than one keratocystic odontogenic tumor, nevoid basal cell carcinoma syndrome is likely. In syndromic patients, the keratocystic odontogenic tumors may arise in the first decade of life. ${ }^{6}$

Keratocystic odontogenic tumors, particularly those that are small, can often be asymptomatic. Larger tumors can cause symptoms with pain and may be associated with tooth root resorption. Although the majority of lesions occur in the posterior aspect of the mandible, those that involve with the maxilla can involve the maxillary sinus and a diagnostic pitfall in the diagnosis of keratocystic odontogenic tumor is its presentation as a cyst in the maxilla. Tumors in maxillary locations can extend into the sinuses, and these maxillary lesions may form cysts that can mimic mucoceles clinically and radiographically. Any cyst in the maxillary sinus that has what appears to be a 'metaplastic' squamous lining should also be examined closely for histologic changes of keratocystic odontogenic tumor. When keratocystic odontogenic tumor occurs in the maxilla, the histology is the same as in other locations. ${ }^{10,11}$

Key histologic criteria for the pathologist to observe in keratocystic odontogenic tumor include the following: (1) a squamous lined cyst with a palisaded basal layer; (2) parakeratosis; (3) keratin production; (4) corrugated surface. This specific combination of findings is never found in dentigerous cysts, ameloblastomas or periapical (radicular) cysts.

The first two findings are the most important and consistent. Surface corrugation may be focal and keratin production may be minimal. Figure 2 shows the features of keratocystic odontogenic tumor. The basal layer is distinct and the cell nuclei have an elongated appearance. Parakeratosis is present and this example shows a corrugated surface as well as keratin. Commonly the epithelium detaches cleanly from the underlying stroma, and while not a diagnostic finding, it is sometimes an initial clue at very low microscopic power that the lesion could be a keratocystic odontogenic tumor. Importantly, even when there is separation of the epithelium from its underlying stroma, the histologic features of the epithelium are maintained.

Multiple smaller cysts can be associated (so-called daughter cysts). No matter how many cysts are present, all have the same histologic features. Keratin 


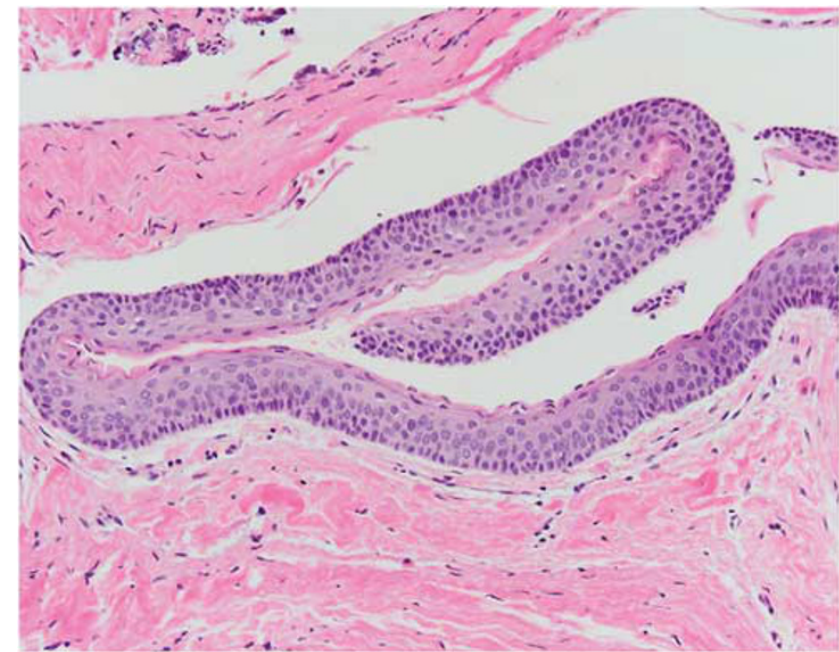

Figure 2 Keratocystic odontogenic tumor. Keratocystic odontogenic tumor has some very distinctive findings that are easily recognized. The squamous epithelial lining of this cystic tumor demonstrates an important finding: a distinct basal layer of cells, which has palisaded architecture. There is parakeratosis, also a critical histologic finding. Note that there is surface corrugation in some areas. Commonly seen in keratocystic odontogenic tumor is the separation of the epithelium from its underlying stroma. Although not a diagnostic feature, this finding is typical of this cystic tumor and its appearance, which can be readily seen at very low microscopic power, should lead to closer examination of the epithelium for findings of keratocystic odontogenic tumor.

production is seen in varying quantities. Keratin can be seen in other odontogenic cysts and thus the other histologic findings must be relied upon.

Keratocystic odontogenic tumor may show inflammation and in that case, the diagnostic features can be lost. However, in most keratocystic odontogenic tumors, at least a focal area showing diagnostic changes can be seen. If just a minimal amount of the classic histologic features of keratocystic odontogenic tumor is seen, even a millimeter in linear extent, then the diagnosis is keratocystic odontogenic tumor. This is a good reason to examine odontogenic cysts in their entirety.

Treatment of keratocystic odontogenic tumor requires (1) complete curettage, (2) often peripheral ostectomy (a procedure where the peripheral bony margins of the cystic cavity are removed/curetted), and (3) treatment with Carnoy's solution, which is usually referred to as chemical cautery. ${ }^{10}$ This solution contains ferric chloride dissolved in ethanol, chloroform, and glacial acetic acid. This solution 'fixes' any residual epithelium that may be left by surgical curetting. Once treated, these patients must be followed for an extended period. Many authorities suggest a panoramic radiograph every year for the first 5 years followed by a panoramic radiograph every other year for the next 5 years. It is clear that this follow-up procedure highlights the potential pervasiveness of this histologically benign process. $^{10}$

\section{Dentigerous cyst}

Dentigerous cysts are the most common of odontogenic cysts and can occur at any tooth location, but most often occur in third molars and maxillary canines, locations most often involved in tooth impaction. ${ }^{12,13}$ Dentigerous cysts are nonkeratinizing cysts that develop in association with the crown of an unerupted or impacted tooth, either primary or permanent. These cysts form most likely from residual remnants of reduced enamel epithelium present after odontogenesis. These remnants of epithelium are attached at the cemento-enamel junction of the tooth, hence the crown of the tooth is contained within the cyst.

Dentigerous cysts usually present clinically as an enlargement of the buccal cortical plate of the maxilla or mandible. They are usually asymptomatic unless they become secondarily inflamed. Dentigerous cysts that are asymptomatic are often identified on radiographs made for other reasons such as orthodontic treatment or when a tooth fails to erupt.

Radiographically dentigerous cysts are associated with the crown of an unerupted tooth and may be unilocular or multilocular. The cyst has a wellcircumscribed border, a finding seen with histologically benign, slow growing processes. The slow pushing growth of dentigerous cysts can deflect teeth, nerves, or other structures, even as the cyst expands to a large size in some cases.

Dentigerous cysts exhibit a non-keratinized squamous lining that is relatively uniform in thickness unless there is associated inflammation. The epithelium is seen to overlie a fibrovascular connective tissue stroma/wall. The epithelium can show a variety of epithelial differentiation including columnar or cuboidal changes (Figure 3). Scattered mucus goblet cell change can be seen focally and this feature can be useful as an ancillary finding to help exclude other squamous epithelial lined cysts, such as keratocystic odontogenic tumor, where mucus cell metaplasia is not seen. Cholesterol clefts and degenerated cellular components are commonly seen in the lumen. This gross appearance can be interpreted by the surgeon as the keratinaceous material that can be present in keratocystic odontogenic tumors. The author has seen numerous pathology specimens sent to the laboratory by the surgeon in which the surgical impression is keratocystic odontogenic tumor because of the keratinaceous appearing material present in a dentigerous cyst. Rarely, other lesions, such as ameloblastoma, squamous carcinoma, or mucoepidermoid carcinoma can arise or be associated with a dentigerous cyst. ${ }^{14-16}$

From a practical standpoint, what is not seen in dentigerous cysts helps separate them from keratocystic odontogenic tumor. Dentigerous cysts have no palisaded basal layer, no parakeratosis, nor corrugated surface, all features present in keratocystic odontogenic tumor. ${ }^{17}$ 


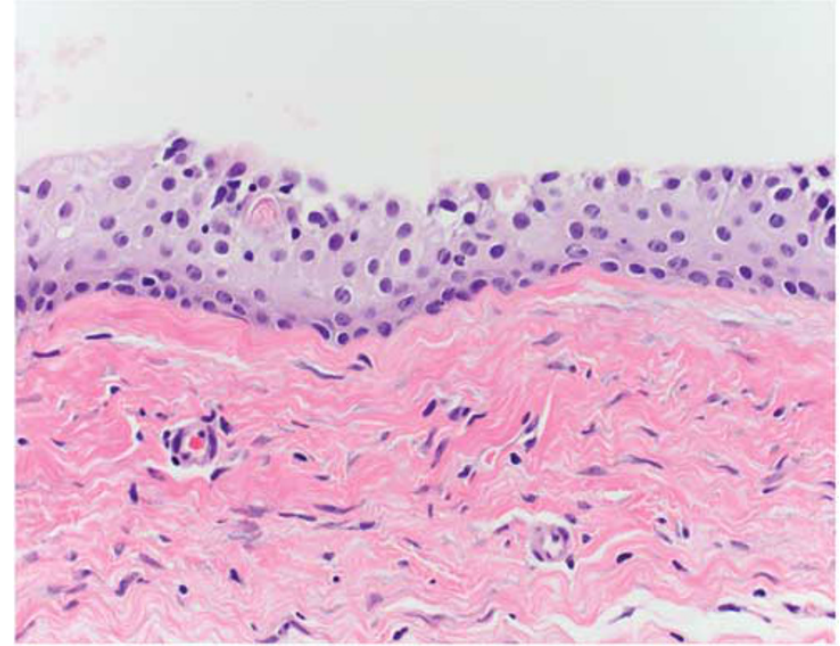

Figure 3 Dentigerous cyst. Dentigerous cysts are among the most common of odontogenic cysts and are characterized by a nonkeratinizing squamous epithelium. Although the epithelium is relatively non-descript, what is not present in the cyst lining is very important in the diagnosis. There is no palisaded basal layer, no parakeratosis, and no surface corrugation as would be seen in keratocystic odontogenic nor reverse polarity of the basal layer or stellate reticulum as seen in ameloblastoma. In some dentigerous cysts, scattered mucus cell or sebaceous cell metaplasia can be seen, findings not seen in keratocystic odontogenic tumor or ameloblastomas. Given the histologic findings of this cyst and if associated with an unerupted tooth, the diagnosis is dentigerous cyst.

The two most common lesions that come in the differential diagnosis include inflamed dentigerous cyst and hyperplastic dental follicle, entities discussed below. ${ }^{18}$ Dentigerous cysts with inflammatory changes can appear identical to radicular cysts but here the radiographic/clinical findings are required for separation. Radicular cysts are periapical lesions (hence an interchangeable diagnostic name, periapical cyst) that are associated with nonvital teeth. Fortunately, the clinical separation of inflamed dentigerous cyst from radicular cyst is easily made with no long-term follow-up or other therapy required.

\section{Radicular cyst}

Radicular cysts are also known as periapical cysts. ${ }^{19,20}$ These cysts arise when embryogenic epithelial remnants, the epithelial rests of Malassez, are induced to proliferate by inflammation/infection of a nonvital (dead) tooth. Most commonly, this is the result of dental caries and resultant inflammation, which invades into the tooth pulp, destroying the neurovascular supply to the tooth. The inflammatory process spreads into the periradicular tissues that include the periodontal ligament (which contains the epithelial rests of Malassez) and the bone that anchors the tooth. It is thought that the inflammatory process leads to the release of mediators that can stimulate these epithelial rests to proliferate. ${ }^{21}$ In some patients, there can be abscess formation at the root tip. In this situation, a sinus may drain from the acute inflammatory component into the intraoral mucosa overlying the cyst. In nonacute situations, either granulation tissue, forming a nodule (periapical granuloma) at the root tip or an epithelial lined cyst with chronic inflammation in the sub-epithelial stroma is seen associated with the root of the nonvital tooth. ${ }^{22}$

Radicular cysts classically present radiographically as a well-demarcated radiolucency directly associated with the root tip. Radicular cysts are generally small, $<1 \mathrm{~cm}$, but can enlarge to many times that size with destructive capabilities because of expansion. ${ }^{23}$

Microscopically radicular cysts show a nonkeratinized stratified squamous epithelium. The epithelium may vary in height from only several cells thick to one in which there is marked hyperplasia. There may be occasional mucus cell metaplasia present (Figure 4). Opposed to keratocystic odontogenic tumors, there are no areas of basal layer palisading, parakeratosis, or surface corrugation. The cyst contents can contain fibrinous material with cholesterol clefts and giant cells. Histologically, radicular cysts can appear identical to dentigerous cysts in cases where there is minimal inflammation in either. Radiographic and clinical correlation are required.

The treatment of radicular cysts can be accomplished with extraction as well as non-surgical and root canal surgical root canal therapy. As with all odontogenic cysts, the entire cyst should be examined histologically, as other processes, including tumors, can appear similar on radiographic evaluation.

\section{Hyperplastic dental follicle}

Hyperplastic dental follicle causes significant trouble to pathologists that are unfamiliar with the diagnosis or concept of the process. Although hyperplastic dental follicle should not be a difficult diagnosis, associated myxoid stroma and odontogenic rests can tempt one to diagnose these developmental lesions incorrectly.

In the normal development of a tooth, a follicle forms around the crown. Occasionally the follicle develops more space around the tooth crown and the follicle appears larger than normal, with the appearance of a small cyst radiographically. ${ }^{24}$ These are designated as hyperplastic dental follicles. Hyperplastic dental follicles often show a myxoid stroma in which can be seen in some cases to be attached to a cuboidal epithelium that represents reduced enamel epithelium. The stroma may also contain small residual odontogenic rests. These small islands of epithelium in a fibromyxoid stroma can be confused with ameloblastic fibroma, although the 


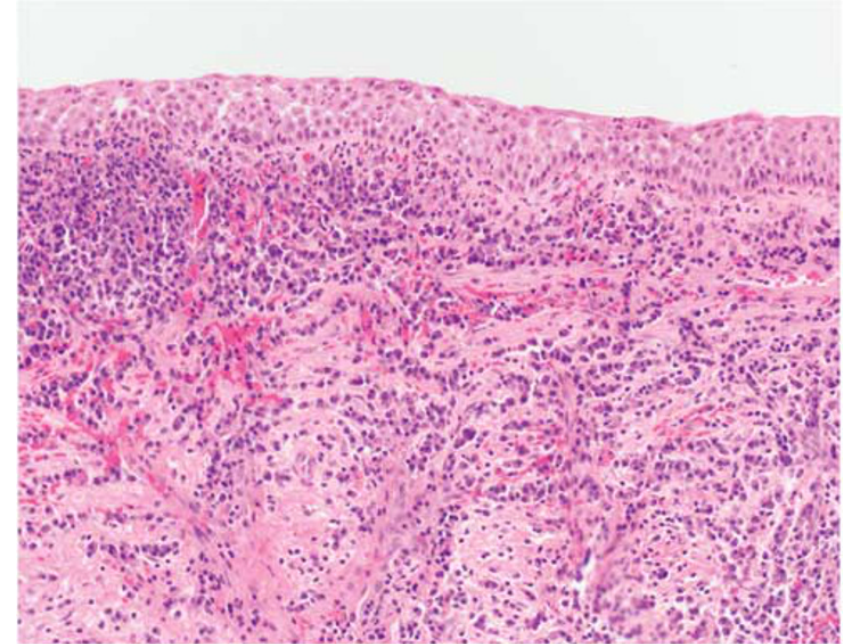

Figure 4 Radicular cyst. Radicular (periapical) cysts are squamouslined cysts. Typically, they show inflammatory cell infiltrates in the wall and in the lining, as seen here. The type of inflammatory cell can be chronic, acute, or a mixture. There may be little inflammation. The radiographic appearance and clinical history are important to the diagnosis of radicular cyst as dentigerous cysts can become inflamed as well and mimic a radicular cyst histologically. Associating a periapical squamous-lined cyst that is inflamed with a carious tooth helps confirm the diagnosis.

clinical and radiographic findings should also help separate these two entities (Figure 5). In some cases, the myxoid stroma can trick the unwary into thinking of myxoma, a neoplasm that has much more myxoid character histologically and importantly, also has a very different clinical and radiographic appearance, but can be superficially similar to dentigerous cyst.

The true differential diagnosis between hyperplastic dental follicle and dentigerous cyst should be made by radiographic correlation. ${ }^{18}$ Most authors suggest that to qualify as a dentigerous cyst, the space between the tooth and the follicle should be $>3-4 \mathrm{~mm}$. However, it is important to realize that the distinction between dentigerous cyst and hyperplastic dental follicle has essentially no consequence and the treatment of each is identical, removal of the tooth and curettage of any cystic lining. No further therapy is required.

\section{Ameloblastomas with cystic change}

Ameloblastomas are neoplasms that usually are not difficult to recognize histologically. ${ }^{25}$ They are composed of stellate reticulum with peripheral basaloid cells showing a palisading arrangement with reverse nuclear polarity. Most commonly we think of ameloblastoma as a solid entity encompassing a number of variants including follicular, acanthomatous, plexiform, granular cell, basal cell, and desmoplastic. Although not as obvious, common components found in ameloblastomas are cystic

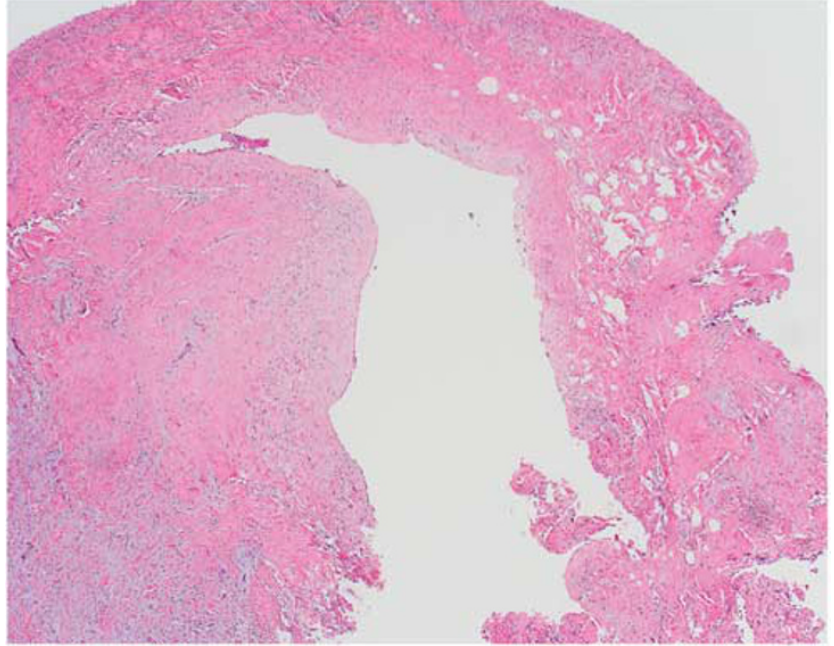

Figure 5 Hyperplastic dental follicle. Hyperplastic dental follicles are composed of fibromyxoid tissue and small epithelial remnants of odontogenesis. In this example, the entire follicle has been removed largely intact with some residual epithelium lining the cyst. Small bits of mineralized material are seen in the wall as well as some isolated epithelial cells. The most problematic issue for general pathologists is confusing them histologically with myxoma or ameloblastic fibroma, both of whose clinical and radiographic findings are entirely different than hyperplastic dental follicle.

areas. In some ameloblastomas, the cystic aspect is most prominent finding, with ameloblastomatous epithelium lining the cyst periphery. An important variant of ameloblastoma is one in which the neoplastic epithelial cells line a cyst with no penetration of the cyst wall by the ameloblastomatous epithelium. This tumor is known as unicystic ameloblastoma. ${ }^{26,27}$ and behaves differently than conventional ameloblastoma. Conversely, conventional ameloblastomas with cystic changes in which the ameloblastomatous epithelium invades the cyst wall behave as conventional ameloblastomas. ${ }^{28,29}$

The cystic lining in unicystic ameloblastoma, as in all ameloblastomas, shows reverse nuclear polarity. Stellate reticulum is also present but can be markedly attenuated. When the lining is thin, unicystic ameloblastoma can be difficult to recognize (Figure 6). The most important finding is that no ameloblastic epithelium is found within the cyst wall. If epithelium is found invading the connective tissue of the cyst wall, the lesion is deemed a conventional ameloblastoma. The treatment of unicystic ameloblastoma is similar to that of keratocystic odontogenic tumor, with complete curettage of the cyst lining and treatment of the residual cavity with Carnoy's solution. ${ }^{28}$ Follow-up is required.

\section{Fibro-osseous lesions}

The term 'fibro-osseous lesion' includes a wide variety of neoplastic and dysplastic processes. It is very important to realize that dysplasia in this setting 


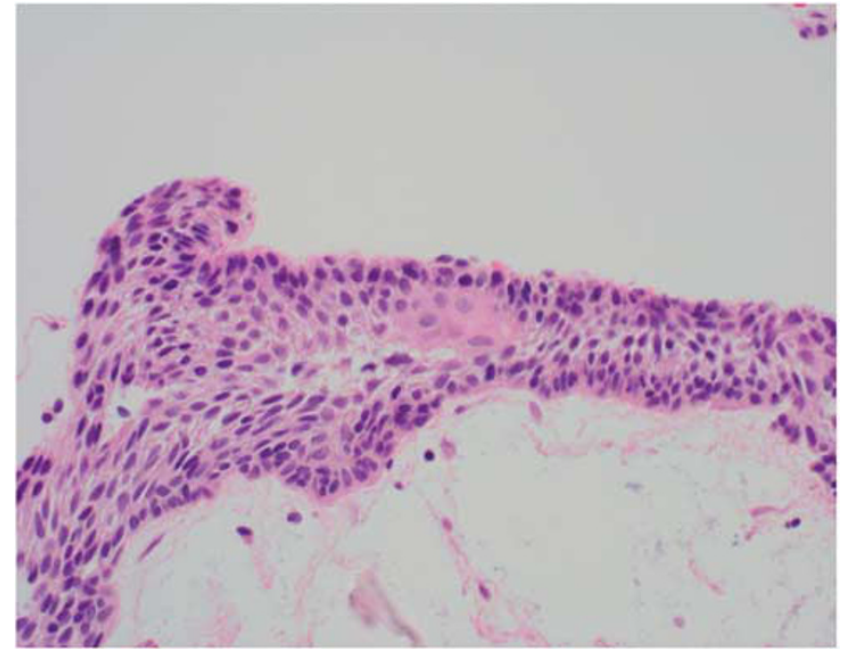

Figure 6 Unicystic ameloblastoma. Unicystic ameloblastoma is an ameloblastoma that is entirely cystic and whose cyst lining is composed of traditional ameloblastomatous components: a palisaded basal layer with reverse nuclear polarity and an overlying stellate reticulum. In this example, squamous change is seen, a common finding in ameloblastoma. Careful examination of such a cystic lesion is required as separation from cystic ameloblastoma is critical from a treatment perspective. Unicystic ameloblastoma has no invasion or penetration of the cyst wall by the ameloblastomatous cystic lining while the similar sounding tumor, cystic ameloblastoma, has an ameloblastomatous cystic lining which also penetrates the wall of the cyst. The former can be treated with thorough curettage of the cyst lining with chemical cautery while the latter could require surgical therapy with excision of margins $1 \mathrm{~cm}$ from the radiographic border of the lesion, the same therapy as for ameloblastoma with no cystic change.

does not mean a pre-neoplastic process but one of disorganized tissue formation.

Diagnosis of odontogenic pathologic processes is frequently enhanced by correlation to the radiographic images. It cannot be over emphasized that in the case of fibro-osseous lesions of the jaws, the radiograph can be the diagnostic discriminator as the histopathology of a number of lesions can appear very similar under the microscope. Pathologists should not attempt to arrive at a final diagnosis without the benefit of a radiographic interpretation. ${ }^{30}$ The two fibro-osseous lesions outlined here include fibrous dysplasia and cementoossifying fibroma.

\section{Fibrous dysplasia}

Fibrous dysplasia is a developmental lesion and characterized histologically by small irregular bone trabeculae that are primarily metaplastic, ie, they appear to arise from the surrounding loose surrounding connective tissue, with no or minimal osteoblastic rimming of trabeculae.

Fibrous dysplasia is associated with an activating mutation at 20q13.2-13.3 in the gene that encodes the alpha subunit of stimulatory $G$ protein $\left[G(s)\right.$ alpha]. ${ }^{31}$
Both women and men are affected equally and there is an equal split between the mandible and maxilla, and classically the disease presents in the second and third decade of life. ${ }^{32}$ Fibrous dysplasia can often be asymptomatic early on but malocclusion of the teeth may cause the patient to seek treatment and in some patients there is obvious malformation of the jaws due to expansion of the bone. ${ }^{33}$ Two forms of the disease can be seen, monostotic, and polyostotic, with the former being by far the most common.

Monostotic fibrous dysplasia makes up about 80$90 \%$ of all cases and as the name implies, involves only a single bone. Interestingly, while mandibular lesions confine themselves to this bone, those occurring in the maxilla can 'drift' to involve other craniofacial bones such as the zygoma, but also the sphenoid, temporal, or even the occipital. This multi-bone involvement of a single lesion is clinically termed craniofacial fibrous dysplasia.

The rare form of the disease is polyostotic, involving multiple bones. Several well-known syndromes are associated with polyostotic fibrous dysplasia, Jaffe-Lichenstein and McCune-Albright. McCune-Albright syndrome is the combination of polyostotic fibrous dysplasia with café-au-lait pigmentation as well as several endocrinopathies. On the other hand, Jaffe-Lichenstein syndrome is the combination of only polyostotic fibrous dysplasia and café-au-lait pigmentation.

The term 'ground glass' is often applied to the plain film's appearance, which is homogeneously radiopaque. ${ }^{34}$ The lesions blend into the surrounding normal bone with an imperceptible border, an important distinction from cemento-ossifying fibroma.

Histologically there are two main components, (1) a loose but cellular background stroma and (2) prominent osseous trabeculae. These trabeculae are irregular in nature but usually uniform in degree of maturation (Figure 7). Although the presence of osteoblastic rimming should not be an overarching feature for which to be searched, there is usually quite minimal osteoblastic rimming of the trabeculae when compared with other entities such as cementoossifying fibroma. A defining feature of fibrous dysplasia is its characteristic fusion with normal bone at the periphery, as noted above, in the radiographic description. ${ }^{35}$

Fibrous dysplasia does not always require therapy. When the bone expansion causes functional or unacceptable cosmetic changes, surgical recontouring of the bone can be performed. In children and adolescents, surgical therapy is sometimes delayed until skeletal maturity is achieved.

\section{Cemento-ossifying fibroma (ossifying fibroma)}

Unlike fibrous dysplasia, which is also considered as a 'fibro-osseous lesion', cemento-ossifying fibroma is 


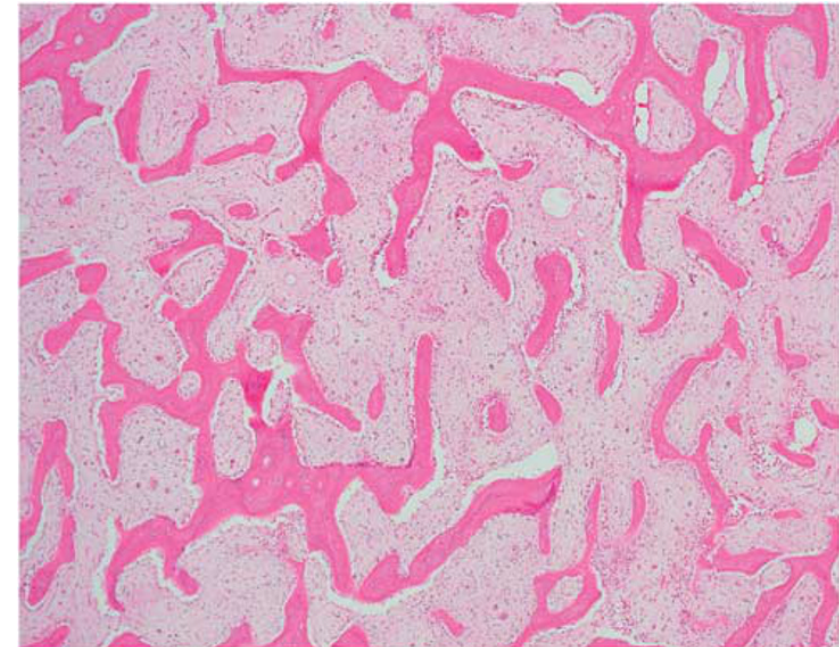

Figure 7 Fibrous dysplasia. The histologic features of fibrous dysplasia are that of bony trabeculae in irregular shapes set in a fibrous stroma. The bone present is usually very uniform in maturation as opposed to that of cemento-ossifying fibroma, where osseous material in various stages of maturation is seen in the same lesion. A very important observation in fibrous dysplasia is the absence of a capsule. Consistent with this not being a neoplastic process, the borders of the lesion fuse into the surrounding bone, both radiographically and histologically.

a neoplasm. ${ }^{36}$ Also unlike fibrous dysplasia, these lesions occur more frequently in women and are three times as likely to occur in the mandible as the maxilla. In an additional separation from fibrous dysplasia, GNAS mutations have not been seen in these tumors. ${ }^{31}$ Further, it is most commonly seen in the fourth decade whereas fibrous dysplasia is usually seen in the second and third decades.

Radiographically these neoplasms have a welldefined contour. They are often radiolucent and some can have homogeneous radiopaque foci. However, they may be completely radiolucent or radiopaque. The opaqueness or lucency is dependent on whether there is mineralization (opaque) or cementum-like material (non-opaque). The most important finding, however, is that the borders of the lesion are corticated, that is, quite distinct. This radiographic finding is unlike fibrous dysplasia where an indistinct margin is seen with the surrounding bone.

From the pathologist's standpoint, the gross findings of excised surgical specimens show a distinct lesion with a smooth periphery, corroborating the finding of a neoplasm. A fragmented specimen at the grossing station is much more in keeping with a fibrous dysplasia, a lesion that must be removed with breaking of the lesion and the firmly attached surrounding normal bone.

Histologically cemento-ossifying fibroma shows some basic features seen in fibrous dysplasia. These are a background fibrous component and bone trabeculae. Unlike fibrous dysplasia, there is a wide range of osteoid, woven bone production, and poorly

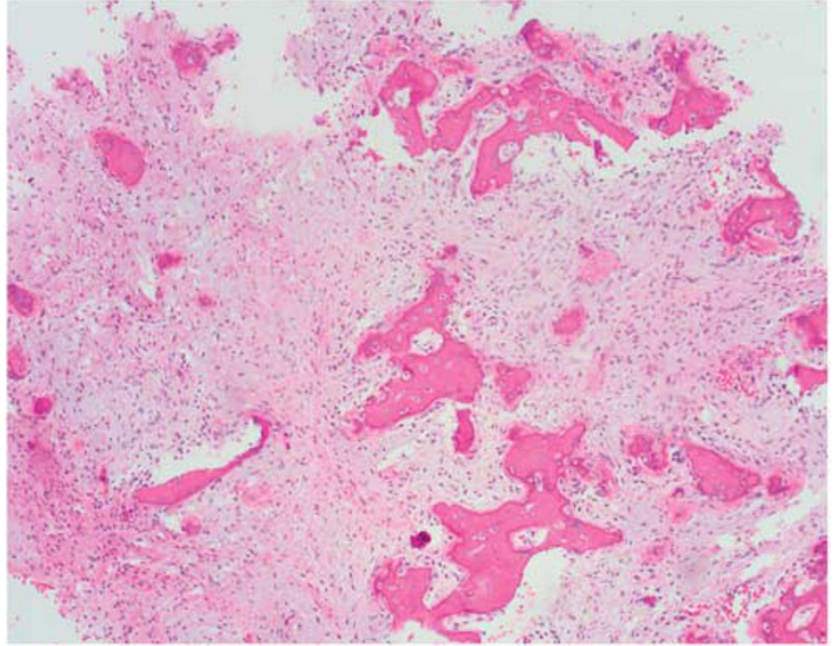

Figure 8 Cemento-ossifying fibroma. As opposed to fibrous dysplasia, cemento-ossifying fibroma is a neoplasm. It has a distinct border/capsule, which is demarcated from the surrounding jaw bone radiographically and histologically. Unlike fibrous dysplasia, there is a wide range of osteoid, woven bone production, and poorly formed trabeculae. In addition, small spherules of eosinophilic material are commonly seen on hematoxylin and eosin stained sections. These small structures of cementum-like material show a peripheral eosinophilic 'brush border' at their periphery.

formed trabeculae. In addition, small spherules of eosinophilic material are commonly seen on hematoxylin and eosin stained sections. These small structures of cementum-like material show a peripheral eosinophilic 'brush border' at their periphery (Figure 8). The distinct presence of cementum-like material admixed with osseous tissue correlates with an odontogenic process and this unique mix of tissue provides a histologic appearance that is very unlike fibrous dysplasia. Cemento-ossifying fibroma is treated by enucleation and curettage. A relatively few, $<5 \%$, of these tumors recur.

\section{Acknowledgments}

This study was supported by the Department of Pathology, University of Iowa

\section{Disclosure/conflict of interest}

The author declares no conflict of interest.

\section{References}

1 Robinson RA, Vincent SD. Tumors and Cysts of the Jaws, AFIP Atlas of Tumor Pathology, Series 4. American Registry of Pathology: Silver Spring, MD, 2012, p 11.

2 Gonzalez-Alva P, Tanaka A, Oku Y, et al. Keratocystic odontogenic tumor: a retrospective study of 183 cases. J Oral Sci 2008;50:205-212. 
3 Meara JG, Shah S, Li KK, et al. The odontogenic keratocyst: A 20-year clinicopathologic review. Laryngoscope 1998;108:280-283.

4 Agaram NP, Collins BM, Barnes L, et al. Molecular analysis to demonstrate that odontogenic keratocysts are neoplastic. Arch Pathol Lab Med 2004;128: 313-317.

5 Madras J, Lapointe H. Keratocystic odontogenic tumour: reclassification of the odontogenic keratocyst from cyst to tumour. J Can Dent Assoc 2008;74: 165-165h.

6 Bresler SC, Padwa BL, Granter SR. Nevoid basal cell carcinoma syndrome (Gorlin Syndrome). Head Neck Pathol 2016;10:119-124.

7 Kimonis VE, Goldstein AM, Pastakia B, et al. Clinical manifestations in 105 persons with nevoid basal cell carcinoma syndrome. Am J Med Genet 1997;69: 299-308.

8 Manfredi M, Vescovi P, Bonanini M, et al. Nevoid basal cell carcinoma syndrome: a review of the literature. Int J Oral Maxillofac Surg 2004;33:117-124.

9 Guo YY, Zhang JY, Li XF, et al. PTCH1 Gene mutations in keratocystic odontogenic tumors: a study of 43 Chinese patients and a systematic review. PLoS One 2013;8:1-9.

10 Myoung $\mathrm{H}$, Hong SP, Hong SD, et al. Odontogenic keratocyst: review of 256 cases for recurrence and clinicopathologic parameters. Oral Surg Oral Med Oral Pathol Oral Radiol Endod 2001;91:328-333.

11 Press SG. Odontogenic tumors of the maxillary sinus. Curr Opin Otolaryngol Head Neck Surg 2008;16:47-54.

12 Shear M. Developmental odontogenic cysts. An update. J Oral Pathol Med 1994;23:1-11.

13 Scholl RJ, Kellett HM, Neumann DP, et al. Cysts and cystic lesions of the mandible: clinical and radiologichistopathologic review. Radiographics. 1999;19: 1107-1124.

14 McMillan MD, Smillie AC. Ameloblastomas associated with dentigerous cysts. Oral Surg Oral Med Oral Pathol 1981;51:489-496.

15 Chretien PB, Carpenter DF, While NS, et al. Squamous carcinoma arising in a dentigerous cyst. Presentation of a fatal case and review of four previously reported cases. Oral Surg Oral Med Oral Pathol 1970;30:809-816.

16 Waldron CA, Koh ML. Central mucoepidermoid carcinoma of the jaws: report of four cases with analysis of the literature and discussion of the relationship to mucoepidermoid, sialo-odontogenic and glandular odontogenic cysts. J Oral Maxillfac Surg 1990;48: 871-877.

17 Dunsche A, Babendererde O, Luttges J et al. Dentigerous cyst versus unicystic ameloblastoma-differential diagnosis in routine histology. J Oral Pathol Med 2003;32:486-491.

18 Daley TD, Wysocki GP. The small dentigerous cyst. A diagnostic dilemma. Oral Surg Oral Med Oral Pathol Oral Radiol Endod 1995;79:77-81.
19 Savage NW, Adkins KF, Weir AV, et al. An histological study of cystic lesions following pulp therapy in deciduous molars. J Oral Pathol 1986;15:209-212.

20 Stockdale CR, Chandler NP. The nature of the periapical lesion-a review of 1108 cases. J Dent 1988;16: 123-129.

21 Browne RM. Some observations on the fluids of odontogenic cysts. J Oral Pathol 1976;5:74-87.

22 Mass E, Kaplan I, Hirshberg A. A clinical and histopathological study of radicular cysts associated with primary molars. J Oral Pathol Med 1995;24: 458-461.

23 Marmary Y, Kutiner G. A radiographic survey of periapical jawbone lesions. Oral Surg Oral Med Oral Pathol 1986;61:405-408.

24 Gardner DG. The central odontogenic fibroma: an attempt at clarification. Oral Surg Oral Med Oral Pathol 1980;50:425-432.

25 Reichart PA, Philipsen HP, Sonner S. Ameloblastoma: biological profile of 3677 cases. Eur J Cancer B Oral Oncol 1995;31B:86-99.

26 Philipsen HP, Reichart PA. Unicystic ameloblastoma. A review of 193 cases from the literature. Oral Oncol 1998;34:317-325.

$27 \mathrm{Li} \mathrm{TJ}$, Kitano M, Arimura K, et al. Recurrence of unicystic ameloblastoma: a case report and review of the literature. Arch Pathol Lab Med 1998;122:371-374.

28 Lee PK, Samman N, Ng IO. Unicystic ameloblastomause of carnoy's solution after enucleation. Int J Oral Maxillofac Surg 2004;33:263-267.

29 Ackermann GL, Altini M, Shear M. The unicystic ameloblastoma: a clinicopathological study of 57 cases. J Oral Pathol 1988;17:541-546.

30 MacDonald-Jankowski DS. Fibro-osseous lesions of the face and jaws. Clin Radiol 2004;59:11-25.

31 Patel MM, Wilkey JF, Abdelsayed R, et al. Analysis of GNAS mutations in cemento-ossifying fibromas and cemento-osseous dysplasias of the jaws. Oral Surg Oral Med Oral Pathol Oral Radiol Endod 2010;109:739-743.

32 Alsharif MJ, Sun ZJ, Chen XM, et al. Benign fibroosseous lesions of the jaws: a study of 127 Chinese patients and review of the literature. Int J Surg Pathol 2009;17:122-134.

33 DiCaprio MR, Enneking WF. Fibrous dysplasia pathophysiology, evaluation, and treatment . J Bone Joint Surg Am 2005;87:1848-1864.

34 Bernaerts A, Vanhoenacker FM, Hintjens J, et al. Tumors and tumor-like lesions of the jaw mixed and radiopaque lesions. JBR-BTR 2006;89:91-99.

35 Regezi JA. Odontogenic cysts, odontogenic tumors, fibro-osseous, and giant cell lesions of the jaws. Mod Pathol 2002;15:331-341.

36 MacDonald-Jankowski DS. Ossifying fibroma: a systematic review. Dento Maxillofac Radiol 2009;38: 495-513. 